\title{
BiGlobal stability analysis in curvilinear coordinates of massively separated lifting bodies
}

\author{
Vassili Kitsios , Daniel Rodríguez, Vassilis Theofilis, Andrew Ooi , Julio Soria \\ Waiter Bassett Aerodymamics Laboratony, Department of Mechanical Engineering, University of Methourne, Victoria 3010, Austratia \\ Laboratoire d'Etudes Aérodynamiques (LEA), Université de Poitiers, CNRS, ENSMA, CEAT, 43 route de l'aérodrome, 86036 Poitiers, France \\ School of Aeronautics, Universidad Politécnica de Madrid, Pża. Cardenal Cisneros 3, E-28040 Madrid, Spain \\ Laboratory For Turbulence Research in Aerospace and Combustion. Department of Mechanical and Aerospace Engineering, Monash University. \\ 3800 Meibourne, Australia
}

Keywords:

Biclobal

Stability

Ailfoil

Ellipse

Separation

Conformal mapping

\begin{abstract}
A B S T R A C T
A methodology based on spectral collocation numerical methods for global flow stability analysis of incompressible external flows is presented. A potential shortcoming of spectral methods, namely the handling of the complex geometries encountered in global stability analysis, has been dealt with successfully in past works by the development of spectral-element methods on unstructured meshes. The present contribution shows that a certain degree of regularity of the geometry may be exploited in order to build a global stability analysis approach based on a regular spectral rectangular grid in curvilinear coordinates and conformal mappings. The derivation of the stability linear operator in curvilinear coordinates is presented along with the discretisation method. Unlike common practice to the solution of the same problem, the matrix discretising the eigenvalue problem is formed and stored. Subspace iteration and massive parallelisation are used in order to recover a wide window of its leading Ritz system. The method is applied to two external flows, both of which are lifting bodies with separation occurring just downstream of the leading edge. Specifically the flow configurations are a NACA 0015 airfoil, and an ellipse of aspect ratio 8 chosen to closely approximate the geometry of the airfoil. Both flow configurations are at an angle of attack of $18^{\circ}$ with a Reynolds number based on the chord length of 200 . The results of the stability analysis for both geometries are presented and illustrate analogous features.
\end{abstract}

\section{Introduction}

While linear stability analysis of canonical, one-dimensional (1D) basic states could utilise basic states provided analytically or recovered with limited numerical effort as solutions of ordinary-differential equations [9,33], analysis of hydrodynamic (and aeroacoustic) instabilities of flows through or over complex geometries invariably relies on large-scale computation. No attempt will be made to review here the wide body of recent literature on the subject of global flow instability, in its flavor of linear instability of essentially nonparallel flows [36] considered herein. The interested reader is referred to the early applications of the theory to the study of canonical problems $[35,17,18,39]$, more recent reviews of the subject $[36,6,32]$, and recent literature on applications of the theory to such diverse applications as non-Newtonian flows [11], rocket-motor engines [4], roughness-induced instability in boundary layers [29], buffeting on aircraft wings [8,7], and configu- 
rations of physiological origin [34]. Global stability modes have also been utilised to augment reduced order models (ROM) constructed using a basis of proper orthogonal decomposition (POD) modes. It has been shown that the addition of linear stability modes to the basis used to create the POD ROM is required to replicate the correct growth rates when the flow transition from a steady to an unsteady state [24,26]. In addition, the maturing of theoretical tools for the control of (canonical) laminar and turbulent flows [15,32] has naturally given rise to interest in applying such tools to control flows in realistic geometries. An integral part of state-of-the-art approaches in this context $[1,12,10]$ is access to a large part of the global (direct and adjoint) eigenspectra (see e.g. [37] in this respect). The present contribution is thus doubly motivated by interest in complex geometries of aeronautical interest and means of provision of access to a large part of the eigenspectium.

The flow configurations of interest presently are an ellipse with an aspect ratio $A_{R}=8$ and a NACA 0015 airfoil, both at an angle of attack of $\alpha=18^{\circ}$ to the freestream. The $A_{R}$ of the ellipse was selected to approximate the airfoil surface as closely as possible, so as to serve as a sense check for the final results. In both cases the Reynolds number $R e_{r} \equiv u_{\infty} c / v=200$, where $u_{\infty}$ is the freestream velocity, $c$ the chord length, and $v$ the kinematic viscosity. For both geometries the flow is incompressible, laminar and steady at $R e_{c}=200$ and becomes unsteady at some point before $R e_{c}=300$. A preliminary version of the present analysis has been presented elsewhere [16]. Here, the steady base flow is a requirement for the formulation of the present stability analysis, leaving a discussion of corrections needed to be made if an unsteady base flow is to be analysed for future work.

It is advantageous to use spectral numerical methods for the stability analysis as they are, at the same level of numerical effort, more accurate than standard finite volume or finite-element alternatives [28]. The disadvantage with classic spectra] collocation methods is the difficulty in handling geometry [3]. While spectrally accurate and geometrically flexible methods exist, based on the spectral/hp-element concept [14], this paper will present a means of undertaking a fluid mechanical stability analysis using spectral collocation numerical methods on a rectangular grid and conformal mapping techniques to represent the geometry. An analogous approach was also employed for the inviscid BiGlobal stability analysis of a skewed planar channel and a planar disc geometry in [19], where the mappings from the physical geometry to the calculation domain were numerically determined. The conformal mapping approach adopted in the present study is more closely related to the work of [1] and [10] where the mapping functions were analytical. The flow configuration in these previous studies were semi-bounded domains, whilst in the present case the flow configurations are lifting bodies.

The paper is organised as follows. In Section 2 the numerical method for the stability calculation will be outlined including: the derivation of the perturbation form of the Navier-Stokes equations (NSE) in both the physical and curvilinear domain; the derivation and discretisation of the stability linear operator in curvilinear coordinates; and the paralle] procedure for solving the resulting eigenvalue problem (EVP). Following this in Section 3 details regarding the generation of both the ellipse and airfoil base flows are presented. In Section $\mathbf{4}$ a general method for transforming the geometry and velocity components between coordinate systems is detailed. The specific conformal mapping functions required to map both the ellipse and airfoil base flows are also provided. All of the derivatives required for the conformal mapping of the ellipse can be derived analytically. However, owing to the complexity of the airfoil conformal mapping function, one resorts to calculating the required derivatives using a symbolic mathematical package. A test function indicative of the base flow is used to verify the airfoil conformal mapping procedure. This same test function is then used in the Section 5 to minimise the error associated with the interpolation of the base flow from the airfoil finite volume grid to the BiGlobal spectral grid. In both cases the errors introduced by the present procedure and the convergence of residuals are quantified. Results of the stability analysis of both flow configurations are finally presented in Section 6 .

\section{Numerical method}

The adopted approach for the stability analysis is to solve an EVP for the linearised perturbation form of the NSE. This will determine if the instabilities are to grow or decay for a given steady laminar base flow. The equations pertaining to the stability calculations are written in curvilinear coordinates so that the physical geometry can be represented whilst undertaking the calculation on a rectangular grid. The approach is illustrated with application to the airfoil geometry in Fig. 1. The domain associated with the finite volume grid used to produce the base flow is coloured grey, and the stability domain is coloured black. Both of these domains are illustrated in the physical space, an intermediary cylindrical space, and the stability calculation domain in Fig. 1(a)-(c), respectively. Performing the stability calculation on a structured rectangular grid, enables the use of efficient spectral numerical methods. The derivation of the perturbation form of the NSE in both physical and curvilinear coordinates is first presented. The discretisation of the linear stability operator in curvilinear coordinates is then detailed, and the parallel procedure for solving the resulting EVP outlined.

\subsection{Perturbation form of the Navier-Stokes equations}

Modal flow instability is studied by perturbing the NSE to determine whether small-amplitude perturbations have a tendency to either grow (be unstable) or decay (be stable) in time (or space). For a steady base flow the decomposition

$$
\mathbf{u}(\mathbf{x}, t)=\overline{\mathbf{u}}(\mathbf{x})+\varepsilon \dot{\mathbf{u}}(\mathbf{x}, t)
$$

can be adopted where $\overline{\mathbf{u}}(\mathbf{x})$ is the steady base flow, $\tilde{\mathbf{u}}(\mathbf{x}, t)$ the perturbation and $\varepsilon \ll 1$ an amplitude parameter, considered small for linearisation. An analogous expression is also adopted for the pressure field $\mathbf{p}(\mathbf{x}, \mathfrak{t )}$. Note that the bold face will be 


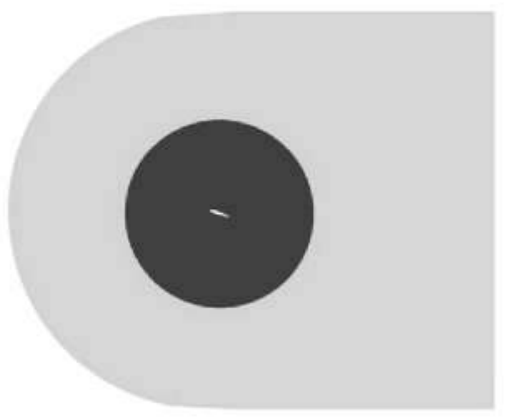

(a)

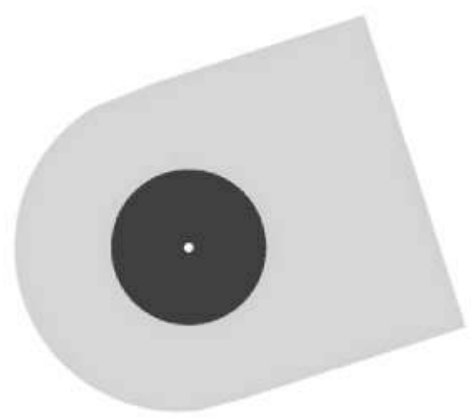

(b)

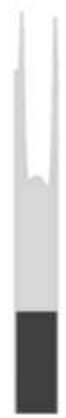

(c)

Fig. 1. Coordinate transformations of the finite volume grid (grey) and the stability grid (black) from the (a) physical coordinates ( $x, y$ ); to the (b) cylindrical space $\left(x_{\text {cyl }}, y_{\text {cyl }}\right)$; and finally to the $(c)$ curvilinear coordinates $\left(\zeta_{1}, \zeta_{2}\right)$.

used throughout to denote vector quantities and matrices. The perturbation form of the NSE is then derived as follows. Firstly the above decomposition is substituted into the viscous incompressible form of the NSE and the terms are expanded. For a steady base flow the NSE as a function of only the steady quantities is also satisfied, and this system of equations is subtracted away from the previous system to produce the perturbation form of the NSE in physical space given by

$$
\begin{aligned}
& \frac{\partial \tilde{u}_{i}}{\partial x_{i}}=0, \text { and } \\
& \frac{\partial \tilde{u}_{i}}{\partial t}+\bar{u}_{j} \frac{\partial \tilde{u}_{i}}{\partial x_{j}}+\tilde{u}_{j} \frac{\partial \bar{u}_{i}}{\partial x_{j}}+\frac{\partial \tilde{p}}{\partial x_{i}}-\frac{1}{R e} \frac{\partial^{2} \tilde{u}_{i}}{\partial x_{j}^{2}}=-\tilde{u}_{j} \frac{\partial \tilde{u}_{i}}{\partial x_{j}} .
\end{aligned}
$$

During the early stages of growth of the instabilities, their amplitude is small and it is valid to assume the nonlinear effects are also small. In this situation, it is acceptable to remove the right-hand-side term $-\tilde{u}_{j} \partial \tilde{u}_{i} / \partial x_{j}$ which produces the perturbation form of the Linearised Navier-Stokes Equations (LNSE). This enables an eigenvalue analysis of the system, which describes the properties of the perturbation for a given base flow $\overline{\mathbf{u}}(\mathbf{x})$. The generation of $\overline{\mathbf{u}}(\mathbf{x})$ for the ellipse and airfoil flow configurations is discussed in Section 3.

The above system of equations is valid if the stability calculation is undertaken in physical space, modifications are required to perform the stability calculation on a rectangular grid whilst still representing the physical geometry. This is done by rewriting this system of equations in curvilinear coordinates producing

$$
\begin{aligned}
& \frac{\partial \tilde{\xi}_{i}}{\partial t}+\frac{1}{h_{j}} \bar{\xi}_{j} \frac{\partial \tilde{\xi}_{i}}{\partial \zeta_{j}}+\frac{1}{h_{j}} \tilde{\xi}_{j} \frac{\partial \bar{\xi}_{i}}{\partial \zeta_{j}}+\frac{1}{h_{i}} \frac{\partial \tilde{p}}{\partial \zeta_{i}}-\frac{1}{R e h_{1} h_{2} h_{3}}\left[\frac{\partial}{\partial \zeta_{1}}\left(\frac{h_{2} h_{3}}{h_{1}} \frac{\partial \tilde{\xi}_{i}}{\partial \zeta_{1}}\right)+\frac{\partial}{\partial \zeta_{2}}\left(\frac{h_{1} h_{3}}{h_{2}} \frac{\partial \tilde{\xi}_{i}}{\partial \zeta_{2}}\right)+\frac{\partial}{\partial \zeta_{3}}\left(\frac{h_{1} h_{2}}{h_{3}} \frac{\partial \tilde{\xi}_{i}}{\partial \zeta_{3}}\right)\right] \\
& =-\frac{1}{h_{j}} \tilde{\xi}_{j} \frac{\partial \tilde{\xi}_{i}}{\partial \xi_{j}} \text {, and } \\
& \frac{\partial}{\partial \zeta_{1}}\left(h_{2} h_{3} \tilde{\xi}_{1}\right)+\frac{\partial}{\partial \zeta_{2}}\left(h_{1} h_{3} \tilde{\xi}_{2}\right)+\frac{\partial}{\partial \zeta_{3}}\left(h_{1} h_{2} \tilde{\xi}_{3}\right)=0 .
\end{aligned}
$$

where $\zeta$ are the coordinates in the calculation domain, and $\xi(\zeta, t)$. These equations are expanded in Appendix A. The $h_{i}$ terms result from the transformation between coordinate systems. The linearised system of equations in this case is produced by setting $\tilde{\xi}_{j} \partial \tilde{\xi}_{i} / \partial \zeta_{j}=0$, enabling an eigenvalue analysis. This provides the instability properties for the base flow $\bar{\xi}(\zeta)$. The base flow in this instance is mapped from the physical space and interpolated onto the spectral rectangular grid. The mapping procedure and derivation of the $h_{i}$ terms are discussed in Section 4.

\subsection{Linear stability operator in curvilinear coordinates}

The flow configurations analysed within this study have two-dimensional (2D) base flows, therefore, the eigenvectors also take on a 2D form. To assist in the following discussion, a (time-step) instantaneous state vector is defined by $\mathbf{q} \equiv(\xi, p)$. Modal perturbations are introduced using the ansatz

$$
\tilde{\mathbf{q}}(\zeta, t)=\hat{\mathbf{q}}\left(\zeta_{1}, \zeta_{2}\right) e^{\mathrm{i} \beta \zeta_{3}-i \Omega t}+\hat{\mathbf{q}}^{*}\left(\zeta_{1}, \zeta_{2}\right) e^{-i \beta^{*} \zeta_{3}+i \Omega^{*} t},
$$

where the ${ }^{*}$ superscript denotes the complex conjugate operation. The second term on the right-hand-side is the complex conjugate of the first term and is required because $\hat{\mathbf{q}}, \beta$ and $\Omega$ can in general be complex, while $\tilde{\mathbf{q}}$ must be real. Here the temporal framework is considered where the complex frequency $\Omega=\Omega_{\mathrm{r}}+\mathrm{i} \Omega_{\mathrm{i}}$ is the to-be-determined eigenvalue, where $\Omega_{\mathrm{r}}$ and 
$\Omega_{\mathrm{i}}$ are the real and imaginary components, respectively. The structure of the perturbation in the spanwise direction is prescribed using the real wavenumber $\beta=2 \pi / L_{\zeta_{3}}$, where $L_{\zeta_{3}}$ is the spanwise wavelength. Substitution of the ansatz (6) into Eq. (4) and the linearised form of Eq. (5), yields four coupled partial-derivative equations forming an EVP for $\Omega$ such that

$$
\mathbf{A}(\overline{\mathbf{q}}, \beta) \hat{\mathbf{q}}=-\mathrm{i} \Omega \mathbf{B} \hat{\mathbf{q}} .
$$

The elements of the linear operators $\mathbf{A}$ and $\mathbf{B}$ are detailed in Appendix $\mathbf{B}$.

The numerical solution of the EVP in Eq. (7) requires an accurate spatial discretisation of the perturbation eigenvector $\hat{\mathbf{q}}$. The use of spectral methods has become standard in stability analysis, as they have been shown to attain the best balance between precision and memory requirements $[20,22,28]$. Chebyshev-Gauss-Lobatto points $(\eta)$ are used here to discretise the wall normal direction $\left(\zeta_{2}\right)$, via an algebraic mapping which allows clustering of points near the wall according to

$$
\zeta_{2}=1 \frac{1-\eta}{1+s+\eta}
$$

where $l$ and $s$ are the parameters that define the clustering and extension of the discretisation. Fourier collocation is the natural choice to discretise the azimuthal direction. Equally spaced Fourier collocation points are used to discretise the periodic direction $\zeta_{1}$. The extents of the BiGlobal grid in these directions are $0<\zeta_{1}<2 \pi$ and $0<\zeta_{2}<\zeta_{2}^{\max }$.

The EVP is also complemented with adequate boundary conditions for the perturbations. No boundary conditions are prescribed explicitly for the $\zeta_{1}$ direction, as the periodicity is imposed with the selection of the Fourier discretisation. In the other spatial direction, the three components of the velocity perturbation are set equal to zero at the wall, and the compatibility condition for pressure of a zero wall normal gradient is imposed. The properties of the perturbations at the far field boundary are not known a priori, so the amplitude functions are linearly extrapolated from within the domain.

\subsection{Solution process and parallelisation}

Due to the size of the leading dimension ( $N_{\mathrm{LD}}$ ) of the $\mathbf{A}$ and $\mathbf{B}$ matrices, recovery the entire spectrum via the $\mathrm{QZ}$ algorithm [27], which is widely adopted for classic analyses of $1 \mathrm{D}$ basic flows, is inappropriate for this application. The QZ algorithm requires the storage of four matrices whose leading dimension scales with $N_{\mathrm{LD}}^{2}$ and the CPU-time needed scales with $N_{\mathrm{LD}}^{3}$. The Arnoldi algorithm [31] obtains a window of the entire spectrum with severa] orders-of-magnitude less computational effort. A shift-and-invert strategy is used, where the most unstable eigenmodes are assumed to be centered on a specified estimate $\sigma$, and the problem

$$
\text { Ằे }=\mu \dot{\mathbf{q}} .
$$

is solved in place of Eq. (7), where

$$
\begin{aligned}
& \breve{A}=(\mathbf{A}-\sigma \mathbf{B})^{-1} \mathbf{B}, \quad \text { and } \\
& \mu=\frac{1}{-\mathrm{i} \Omega-\sigma} .
\end{aligned}
$$

The structure of B makes it possible to store just one matrix, a modified version of the LU-decomposition of A. More details on the algorithmic procedure can be found in [36]. As the primary focus of this paper is the solution methodology, $\sigma$ is not varied. For all calculations presented within $\sigma=0$. This means that for a given number of Arnoldi iterations, eigenvalues equally spaced from the origin have the same level of accuracy. In future studies $\sigma$ could be exploited to improve convergence of eigenvalues located further from the origin.

Eigenvalue problems of this nature require extremely high resolution in order to attain convergence of the eigenvalue spectrum. It is necessary to store and operate on matrices with $N_{\mathrm{LD}} \approx 10^{5}$, which translates to approximately 390 gigabytes of memory, clearly not possible on a serial-machine. A recently developed parallel EVP solver [30] is adopted herein; it employs optimised dense linear algebra libraries and massive parallelisation to make use of distributed-memory supercomputers. Data distribution makes it possible to store and operate on matrices whose dimension is only a function of the number of processors, so the resolution is constrained only by the number of CPUs available. The work distribution also permits a reduction in wall-time of the computation. There are four main tasks required to solve the EVP. The required CPU-time of each of these tasks is estimated as a function of the number of processors ( $N_{\text {prox }}$ ) and $N_{\mathrm{LD}}$. The first task is the generation and distribution of the matrices amongst the processors. The time required for this process

$$
\mathrm{t}_{\mathrm{EVP}} \propto\left(1+\frac{K_{0}}{N_{\mathrm{proc}}}\right) N_{\mathrm{LD}}^{2}
$$

where $K_{0}$ is a constant of proportionality. The second task is the LU-decomposition and is performed by the ScaLAPACK routine pzgetrf. The estimated time for this task is

$$
\mathrm{t}_{\mathrm{LU}} \propto \frac{N_{\mathrm{LD}}^{3}}{N_{\text {proc }}} \text {. }
$$


The Arnoldi iterations are the third task. This involves the iterative generation of a Krylov subspace and a Hessenberg matrix through a given number of back-substitutions (one per iteration) on the LU-decomposed modified A matrix. Typically the number of iterations $(\mathrm{m})$ used to recover an adequate portion of the spectrum is between 200 and 1000 . The use of a large $m$ also reduces the dependence of the results on the shift parameter $\sigma$. The time associated per iteration of this task is

$$
t_{\mathrm{Ar}} \propto\left(1+K_{1} N_{\mathrm{LD}}+K_{2} N_{\mathrm{LD}} N_{\text {proc }}\right) \frac{N_{\mathrm{LD}}}{N_{\text {proc }}},
$$

where $K_{1}$ and $K_{2}$ are constants of proportionality. The final task is to perform a serial QZ algorithm to recover the Ritz values of $\check{A}$ as the eigenspectrum of the $(m \times m)$ Hessenberg matrix, and the product of $(m \times m)$ and $\left(N_{\mathrm{LD}} \times m\right)$ matrices to compute the associated Ritz vectors. The estimated time taken for this step is

$$
t_{\text {EIG }} \propto N_{\mathrm{LD}}+K_{3} N_{\text {proc }}+K_{4} N_{\text {proc }} N_{\text {LD }}
$$

where $K_{3}$ and $K_{4}$ are constants of proportionality. The code was validated and demonstrated to work satisfactorily on the supercomputers Mare Nostrum (http://www.bsc.es) using up to 1024 processors and Blue Gene/P (http://www.fz-juelich.de) using up to 4096 processors. Further details of the code and scalability tests are presented in [30]. The base flows are the variable-coefficients of the partial-derivative EVP and condition the accuracy of the EVP solution. The generation of these base flows and the coefficients of the EVP will be discussed in some detail below.

\section{Generation of base flows}

The base flows of both the ellipse and airfoil are required inputs for the stability analysis. These base flows are generated in physical space using unstructured grids. The specifics are detailed below.

\subsection{Ellipse base flow}

An ellipse of $A_{R}=8$ is placed at $\alpha=18^{\circ}$ to the oncoming incompressible flow. The 2D flow around this object is calculated using the code ADFC [13]. A time-accurate integration of the equations of motion is performed using a semi-Lagrangian timeintegration scheme. The Taylor-Hood finite-element method is adopted for the spatial discretisation. A rectangular domain is used in which $x / c \in[-10,15] \times y / c \in[-10,10]$, with the center of the ellipse placed at $(x=0, y=0)$. A Dirichlet boundary condition of $\mathbf{u}=\left(u_{\infty}, 0,0\right)$ is applied at the inlet, top, and bottom boundaries. A Neumann boundary condition is applied to the outlet boundary where the velocities derivatives normal to the boundary are set equal to zero. A wall boundary condition is also applied at the ellipse surface. Fig. 2 illustrates instantaneous snapshots of the velocity magnitude at $R e_{c}=200$ and $R e_{c}=300$ in a sub-domain in proximity to the ellipse body. In both instances the flow separates just downstream of the leading edge, with the flow steady at $R e_{c}=200$ and unsteady at $R e_{c}=300$. The present stability formulation is only valid for steady flows, consequently the steady base flow of $R e_{c}=200$ is adopted.

\subsection{Airfoil base flow}

The second flow configuration is a 2D NACA 0015 airfoil, again at $\alpha=18^{\circ}$. The flow field is numerically simulated using the incompressible version of the code CDP. This code was developed at the Stanford Center for Turbulence Research and is an unstructured finite volume based solver. Numerical dissipation is minimised by discretising the continuity and momentum equations such that they discretely conserve kinetic energy. This is enforced on the pressure and convective terms using the approach outlined in [21]. A second-order accurate Adams-Bashforth method is used for the time-integration, and a second-order accurate central difference scheme adapted for unstructured grids is used for the spatial differentiation [21]. A Ctype grid of the airfoil is adopted, with the block topology illustrated in Fig. 3(a). The grid is summarised by the arc ( $\mathscr{C})$ and

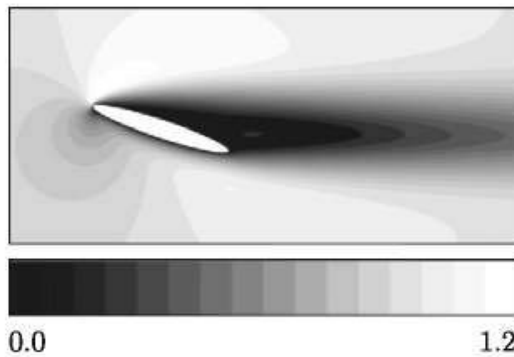

(a)

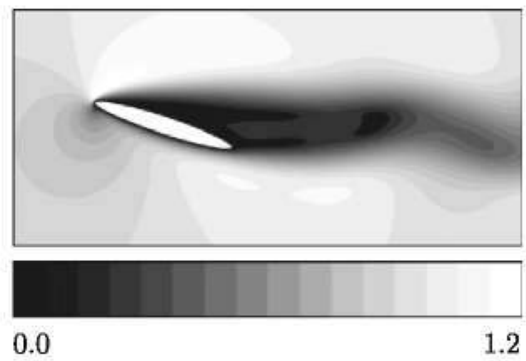

(b)

Fig. 2. Instantaneous velocity magnitude of the ellipse base flow with a $R e_{c}$ of: (a) 200 ; and (b) 300 . 


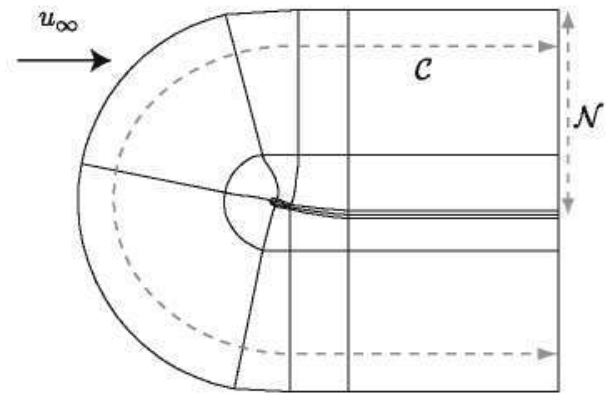

(a)

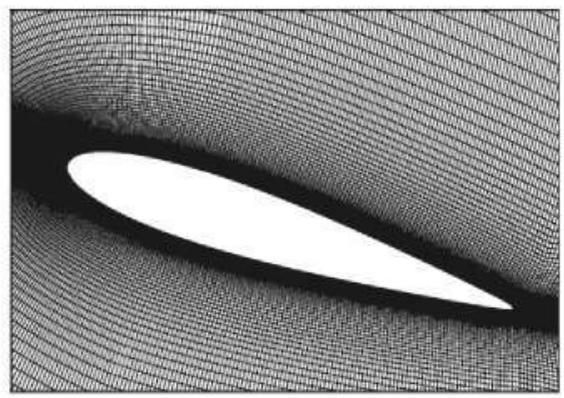

(b)

Fig. 3. The grid used to generate the airfoil base flow, with the: (a) block topology; and (b) mesh at the airfoil boundary surface. Note the domain associated with this grid, is the grey coloured domain in Fig. 1(a).

the dimension between the airfoil body and the outer boundary $(\mathscr{N})$. The computational domain size in these directions is $L_{\mathscr{S}} \times L_{\mathscr{6}}=60 \mathrm{c} \times 10 \mathrm{c}$, and is of comparable size to the previous ellipse grid. The number of cells in the $\mathscr{C}$ and $\mathscr{N}$ directions are $850 \times 120$, respectively, for a total of 102,000 elements. The grid in proximity to the airfoil body is illustrated in Fig. 3(b). The airfoil surface geometry is defined by the conformal mapping process discussed in Section 4.3. This is important as it ensures that the BiGlobal and finite volume grids lie on the same surface. The application of the boundary conditions is consistent with the previous ellipse case. The simulation is advanced in time with a constant time-step size of $\Delta t=2 \times 10^{-4} \mathrm{c} / \mathrm{u}_{\infty}$, ensuring that the maximum Courant Friedrichs Lewy (CFL) number in the entire domain does not exceed 1.0 . The simulation is run until a laminar steady state is attained, which occurs after a time of $40 \mathrm{c} / \mathrm{u}_{\infty}$ non-dimensional units. Consistent with the ellipse, the flow separates just downstream of the leading edge and is steady at $R e_{c}=200$ (see Fig. $4(\mathrm{a})$ ) and unsteady at $\operatorname{Re}_{c}=300$ (see Fig. 4(b)).

\section{Conformal mapping between physical and stability calculation domains}

Both base flows are generated in physical space and they must be transformed into the curvilinear domain before performing the stability calculation. The general curvilinear coordinate system is first discussed and the specific mapping functions for both configurations are then presented. The ellipse transformation is simple enough to derive the required analytical derivatives by hand, whereas one resorts to using a symbolic mathematics package for the airfoil case. The airfoil mapping process is then validated using a test function indicative of the base flow.

\subsection{Curvilinear coordinate system}

The following discussion documents the transformation between two orthogonal coordinate systems: the physical one; and the curvilinear coordinate system in which the stability analysis is undertaken. The physical coordinate system is $\mathbf{x}=x \mathbf{j}_{1}+y \mathbf{j}_{2}+z \mathbf{j}_{3}$ with unit vectors $\mathbf{j}_{1}, \mathbf{j}_{2}$ and $\mathbf{j}_{3}$, and the curvilinear coordinate system is $\zeta_{2} \zeta_{1} \mathbf{a}_{1}+\zeta_{2} \mathbf{a}_{2}+\zeta_{3} \mathbf{a}_{3}$ with unit vectors $\mathbf{a}_{1}, \mathbf{a}_{2}$ and $\mathbf{a}_{3}$. Given that the conformal mapping functions $\mathbf{x}(\zeta)$ are all available and differentiable, the basis of the curvilinear coordinate system is determined by

$$
\mathbf{a}_{k}=\frac{\mathbf{e}_{k}}{h_{k}}, \quad \text { where }
$$

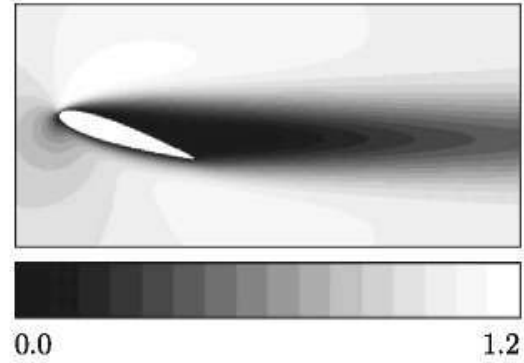

(a)

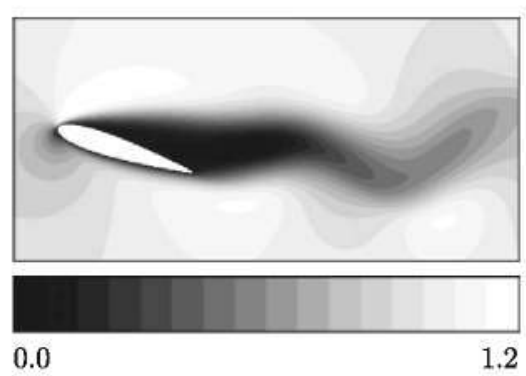

(b)

Fig. 4. Instantaneous velocity magnitude of the airfoil base flow with a $\operatorname{Re}_{\mathrm{c}}$ of: (a) 200 ; and (b) 300 . 


$$
\begin{aligned}
& \mathbf{e}_{k}=\left(\frac{\partial x}{\partial \zeta_{k}}, \frac{\partial y}{\partial \zeta_{k}}, \frac{\partial z}{\partial \zeta_{k}}\right), \text { and } \\
& h_{k}=\left\|\mathbf{e}_{k}\right\|=\sqrt{\left(\frac{\partial x}{\partial \zeta_{k}}\right)^{2}+\left(\frac{\partial y}{\partial \zeta_{k}}\right)^{2}+\left(\frac{\partial z}{\partial \zeta_{k}}\right)^{2}},
\end{aligned}
$$

where $h_{k}$ are the shape factors ([23]) first mentioned in Section 2.1 . The physical velocity field $\mathbf{u}=u \mathbf{j}_{1}+v \mathbf{j}_{2}+w \mathbf{j}_{3}$ can then be projected onto the curvilinear basis to produce the velocity field $\xi=\xi_{1} \mathbf{a}_{1}+\xi_{2} \mathbf{a}_{2}+\xi_{3} \mathbf{a}_{3}$, where each component $\xi_{k}$ is given by

$$
\check{\check{c}}_{k}=u\left(\mathbf{j}_{1} \cdot \mathbf{a}_{k}\right)+v\left(\mathbf{j}_{2} \cdot \mathbf{a}_{k}\right)+w\left(\mathbf{j}_{3} \cdot \mathbf{a}_{k}\right)=u \frac{1}{h_{k}} \frac{\partial x}{\partial \zeta_{k}}+v \frac{1}{h_{k}} \frac{\partial y}{\partial \zeta_{k}}+w \frac{1}{h_{k}} \frac{\partial z}{\partial \zeta_{k}} .
$$

The specific conformal mapping functions transforming the ellipse and airfoil to the rectangular calculation domain are now presented. For both geometries the third component is unchanged between each of the mappings. This means that $z=\zeta_{3}$, and by consequence $\partial z / \partial \zeta_{3}=\partial \zeta_{3} / \partial z=1, h_{3}=1$ and all derivatives of $h_{3}$ are zero. The following discussion will, therefore, be confined to the $(x, y)$ and $\left(\zeta_{1}, \zeta_{2}\right)$ planes.

\subsection{Ellipse conformal mapping}

The conformal mapping functions for the ellipse are

$$
\begin{aligned}
& x=\eta^{\prime} \cosh \left(\zeta_{2}\right) \cos \left(\zeta_{1}\right), \quad \text { and } \\
& y=\vartheta \sinh \left(\zeta_{2}\right) \sin \left(\zeta_{1}\right), \text { where } \\
& y=c \sqrt{1-A_{R}^{2}} .
\end{aligned}
$$

The shape factors for the transformation of each of the fields discussed previously are determined analytically to be

$$
h_{1}=h_{2}=h=\hat{\jmath} \cdot \sqrt{\sinh ^{2}\left(\zeta_{2}\right)+\sin ^{2}\left(\zeta_{1}\right)} .
$$

The derivatives of these shape factors additionally required for the operator $A$ are

$$
\begin{aligned}
& \frac{\partial h}{\partial \zeta_{1}}=\frac{\hat{\jmath}^{2} \sin \left(2 \zeta_{1}\right)}{2 h}, \text { and } \\
& \frac{\partial h}{\partial \zeta_{2}}=\frac{\hat{\jmath}^{2} \sinh \left(2 \zeta_{2}\right)}{2 h} .
\end{aligned}
$$

All of these terms are analytical and exact, and there is consequently no accumulation of errors associated with the mapping.

\subsection{Airfoil conformal mapping}

The mapping between the curvilinear and physical coordinates for an airfoil geometry is a little more involved: in total there are four mappings required. The first, maps from curvilinear space $\left(\zeta_{1}, \zeta_{2}\right)$ to an intermediary cylindrical space $\left(x_{c y l}, y_{c y l}\right)$ via

$$
\begin{aligned}
& x_{\mathrm{cyl}}=\left(\zeta_{2}+1\right) \cos \left(\zeta_{1}\right), \text { and } \\
& y_{\mathrm{cyl}}=\left(\zeta_{2}+1\right) \sin \left(\zeta_{1}\right) .
\end{aligned}
$$

A Joukowskì transformation is then used to convert the cylindrical grid to an airfoil shaped mesh of coordinates ( $x_{\text {air }}, y_{\text {air }}$ ) by

$$
\begin{aligned}
& x_{\mathrm{air}}=\left(x_{\mathrm{cyl}}+b\right)\left(1+\frac{\mathfrak{a}^{2}}{\left(x_{\mathrm{cyl}}+b\right)^{2}+y_{\mathrm{cyl}}^{2}}\right), \text { and } \\
& y_{\mathrm{air}}=y_{\mathrm{cyl}}\left(1-\frac{\mathfrak{a}^{2}}{\left(x_{\mathrm{cyl}}+b\right)^{2}+y_{\mathrm{cyl}}^{2}}\right)
\end{aligned}
$$

where the parameters $a$ and $b$ are selected so that the airfoil shape best represents a NACA 0015 airfoil. This airfoil shaped mesh is then translated by $\left(x_{\text {shift }}, y_{\text {shift }}\right)$ and scaled by $L_{\text {siale }}$ according to 


$$
\begin{aligned}
& x_{\text {air scaled }}=L_{\text {scale }}\left(x_{\text {air }}-x_{\text {shiff }}\right), \text { and } \\
& y_{\text {air,scaled }}=L_{\text {scale }}\left(y_{\text {air }}-y_{\text {shift }}\right),
\end{aligned}
$$

and finally rotated clockwise about the leading edge by $\alpha$ to produce the final airfoil coordinates via

$$
\begin{aligned}
& x=x_{\text {air,scaled }} \cos (\alpha)+y_{\text {air,scaled }} \sin (\alpha), \text { and } \\
& y=y_{\text {air,scaled }} \cos (\alpha)-x_{\text {air,scaled }} \sin (\alpha) .
\end{aligned}
$$

The intermediary coordinate systems are then removed through substitution to produce complicated expressions for $x\left(\zeta_{1}, \zeta_{2}\right)$ and $y\left(\zeta_{1}, \zeta_{2}\right)$, from which the shape factor expressions are determined. This enables the stability calculation to be undertaken on the rectangular curvilinear grid, whilst representing the geometry of an airfoil in physical space. To convert the mean velocity field calculated in physical space to the curvilinear coordinates, the inverse transformations are undertaken in the opposite order. The finite volume and BiGlobal grids in each of the three spaces are illustrated in Fig. 1. Differentiation of the airfoil conformal mapping functions by hand is prone to error, so a symbolic mathematics package is used to determine the required expressions. The symbolic expressions are then exported as $\mathrm{C}++$ code and used directly in the conformal mapping program.

\subsection{Validation of the conformal mapping process}

To check the derivative fields of both the ellipse and airfoil mapping functions are determined correctly, 40 analytical relationships akin to

$$
\frac{\partial x_{k}}{\partial \zeta_{1}} \frac{\partial \zeta_{1}}{\partial x_{j}}+\frac{\partial x_{k}}{\partial \zeta_{2}} \frac{\partial \zeta_{2}}{\partial x_{j}}=\delta_{j k}
$$

are evaluated at every point in the domain and produce the expected result, where $\hat{o}_{j k}$ is the delta function.

A procedure to verify the airfoil conformal mapping functions is described next. First, a test function indicative of the base flow is chosen. Then a series of rectangular grids with increasing mesh resolution are generated in curvilinear space, each with $\zeta_{2}^{\max }=1.0$. The cells in each case are of constant size. The geometry in the curvilinear space $\zeta$ is mapped to the physical space $\mathbf{x}$ where the test function is applied generating $\mathbf{u}$ and $\partial \mathbf{u} / \partial \mathbf{x}$. These fields are then mapped into the curvilinear space to produce $\xi$ and $\partial \xi / \partial \zeta$. The velocity derivative fields $\partial \xi / \partial \zeta$ are then approximated by spatially differentiating the velocity field $\xi$ using a finite difference scheme. If the mapping is performed correctly, the difference between the mapped field and the finite difference approximation of the derivative fields should decrease with increasing resolution of the curvilinear grids.

An additional complexity arises in the application of the test function. A test function that mimics a typical base flow is desired, one that is zero at the airfoil surface and grows to the freestream value as a function of the distance from the wall. This can be achieved by setting the velocity components of $\mathbf{u}$ equal to the test function $\Psi$ which is dependent on the associated wall normal curvilinear coordinate $\zeta_{2}$ via

$$
u(x, y)=v(x, y)=\Psi\left(\zeta_{2}\right)=\operatorname{erf}\left(\zeta_{2}\right),
$$

where erf is the error function. As $\mathbf{u}$ is not a function of $\mathbf{x}$ directly, the spatial derivatives of $\boldsymbol{u}$ in airfoil space must be mapped from the spatial derivatives of $\Psi$ in curvilinear space according to

$$
\begin{aligned}
& \frac{\partial u(x, y)}{\partial x_{k}}=\frac{\partial v(x, y)}{\partial x_{k}}=\frac{\partial \Psi\left(\zeta_{2}\right)}{\partial x_{k}}=\frac{\partial \Psi\left(\zeta_{2}\right)}{\partial \zeta_{2}} \frac{\partial \zeta_{2}}{\partial x_{k}}, \text { where } \\
& \frac{\partial \Psi\left(\zeta_{2}\right)}{\partial \zeta_{2}}=\frac{4}{\sqrt{\pi}} e^{-\sqrt{2 \xi_{2}}} .
\end{aligned}
$$

The fields $u$ and $\partial u / \partial y$ are illustrated in Fig. 5(a) and (b), respectively.

The associated velocity and derivative fields are required in curvilinear coordinates. The velocity components $\mathbf{u}$ in the airfoil space are mapped back through the required transformations into the curvilinear space using Eq. (19) producing $\xi$, with $\zeta_{1}$ illustrated in Fig. 5(c). The velocity derivative fields $\partial u_{i} / \partial x_{j}$ are mapped according to

$$
\begin{aligned}
& \frac{\partial \xi_{k}}{\partial \xi_{1}}=\frac{\partial \xi_{k}}{\partial x} \frac{\partial x}{\partial \zeta_{1}}+\frac{\partial \xi_{k}}{\partial y} \frac{\partial y}{\partial \zeta_{1}}, \text { where } \\
& \frac{\partial \xi_{k}}{\partial x_{j}}=\frac{\partial u}{\partial x_{j}} \frac{1}{h_{k}} \frac{\partial x}{\partial \zeta_{k}^{r}}-\mathfrak{u} \frac{1}{h_{k}^{2}} \frac{\partial x_{j}}{\partial \zeta_{k}} \frac{\partial h_{k}}{\partial x}+\frac{\partial v}{\partial x_{j}} \frac{1}{h_{k}} \frac{\partial y}{\partial \zeta_{k}}-v \frac{1}{h_{k}^{2}} \frac{\partial y}{\partial \zeta_{k}} \frac{\partial h_{k}}{\partial x_{j}},
\end{aligned}
$$

with $\partial \xi_{1} / \partial \xi_{2}$ illustrated in Fig. 5(e).

The mapped derivative fields $\partial \xi_{i} / \partial_{j}$ are checked by calculating them from the $\xi$ field using a forth order accurate finite difference method. This is possible because the cells are rectangular in the curvilinear space. The finite difference operation produces the $\partial \xi_{i}^{\text {diff }} / \partial \zeta_{j}$ fields, with $\partial \xi_{1}^{\text {diff }} / \partial \zeta_{2}$ illustrated in Fig. $5(\mathrm{~d})$. The squared difference field between $\partial \xi_{1} / \partial \zeta_{2}$ and $\partial \xi_{1}^{\text {diff }} / \partial \zeta_{2}$ is illustrated in Fig. 5(f). The normalised squared errors associated with the spatial derivative fields of $\xi$ are 

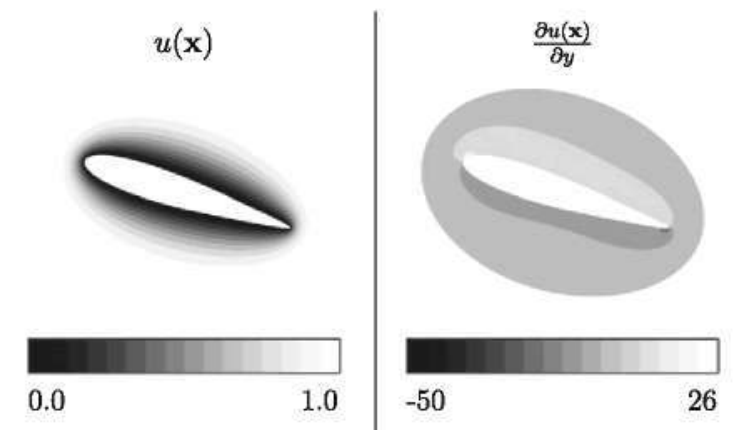

(a)

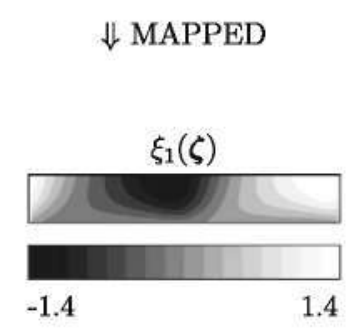

(c)

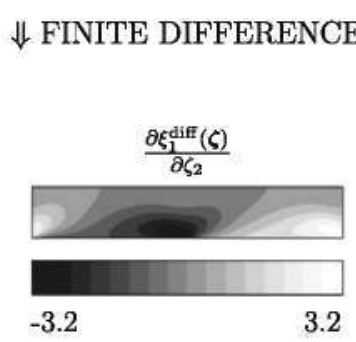

(d)

3.2

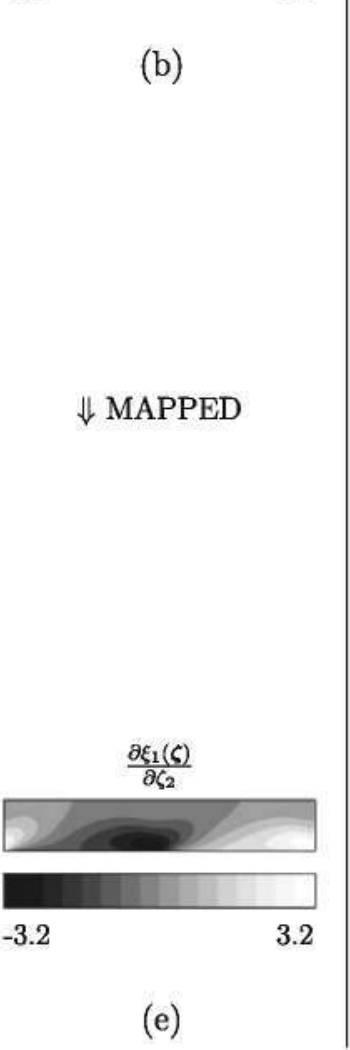

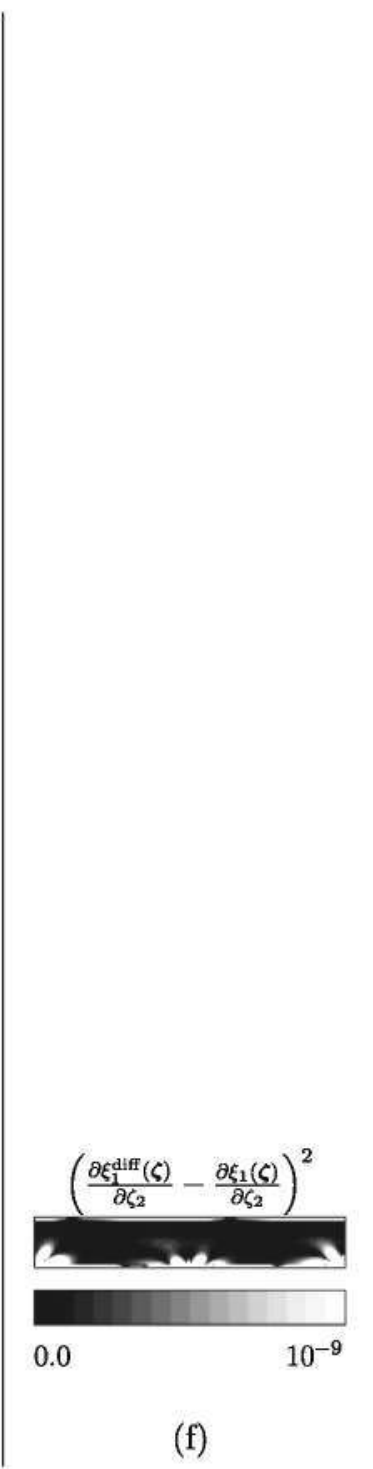

(f)

Fig. 5. Validation of the mapping of the $\partial u(\mathbf{x}) / \partial y$ field. The test function field variables in airfoil space: (a) $u(\mathbf{x})$; and (b) $\partial u(\mathbf{x}) / \partial y$, are mapped to the curvilinear space, respectively producing field variables:(c) $\xi_{1}$; and (e) $\partial \xi_{1} / \partial \zeta_{2}$. The latter field is checked via a finite difference calculation applied to the $\xi_{1}(\zeta)$ field, producing (d) $\partial \xi_{1}^{\text {diff }}(\zeta) / \partial \zeta_{2}$. The discrepancy between the finite difference and mapped derivative fields is quantified by (f) $\left(\partial \xi_{1}^{\text {diff }}(\zeta) / \partial \zeta_{2}-\partial \xi_{1}(\zeta) / \partial \zeta_{2}\right)^{2}$.

$$
\epsilon_{i j}^{\text {map }}=\frac{\int_{V}\left(\partial \xi_{i} / \partial \zeta_{j}-\partial \xi_{i}^{\text {diff }} / \partial \zeta_{j}\right)^{2} d \xi}{\int_{V}\left(\partial \xi_{i} / \partial \zeta_{j}\right)^{2} d \xi}
$$

where $V$ is the domain volume in the curvilinear coordinate system. The mapped derivatives are analytic and their accuracy should not change with resolution of the mesh. The accuracy of the finite difference calculation, however, should improve with increased resolution of the mesh. The mapping approach is, therefore, validated if $\epsilon_{i, j}^{\text {map }}$ decreases with increasing grid resolution. This is the case for all of the error measures with $\epsilon_{1,1}^{\text {map }}$ and $\epsilon_{1,2}^{\text {map }}$ illustrated in Fig. 6. $\epsilon_{1,2}^{\text {map }}$ is consistently larger than $\epsilon_{1,1}^{\mathrm{map}}$ for each mesh because the cell size in the $\zeta_{2}$ direction is slightly larger than in the $\zeta_{1}$ direction, and its finite difference approximation is, therefore, less accurate.

\section{Interpolation of data}

The basic velocity field must be spatially differentiated and interpolated from the original finite volume (FV) or finite-element (FE) grids to the BiGlobal grid, on which the stability analysis will be undertaken. Aside from the selection of the interpolation method, there are two possible options available in which to proceed. The first option is to interpolate and 


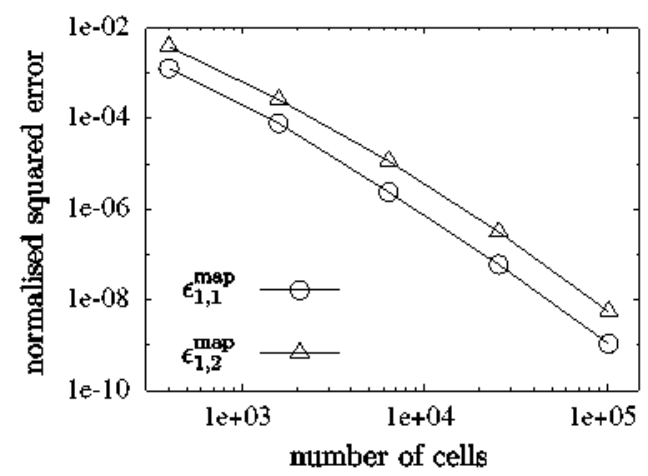

Fig. 6. Squared difference between the mapped and finite difference approximation of the test function derivatives plotted as a function of the number of cells.

differentiate the fields in the physical coordinate system (see Fig. 1(a)) and then map the results to the curvilinear space. The other option is to first map the raw data from the physical to the curvilinear coordinates and undertake the interpolation and spatial differentiation there (see Fig. 1(c)). Even thongh the conformal mapping method is analytical and exact it is also highly non-linear. Small changes in the initial field may well produce drastic changes in the mapped field. It is, therefore, advisable to apply the mapping directly to the raw data and to not contaminate the process with any additional errors that may arise from the interpolation. In addition interpolation processes have a tendency to smooth data and it is preferable to perform this operation last. Both options were tested and as expected the second produced smoother, more consistent surfaces. The details of the second approach are now outlined.

As indicated above, the original basic flow FV/FE fields are first mapped from the physical into the curvilinear coordinate system. A moving least squares polynomial interpolation method is used to interpolate and spatially differentiate the velocity fields from the original FV/FE grid mapped into curvilinear space onto the rectangular BiGlobal grid. A new polynomial surface, is centered at each point on the BiGlobal grid and fit to the velocity field on the original FV/FE grid. This polynomial surface is then analytically differentiated to determine the spatial derivatives at each point. The number of points $N_{p}$, required to define a $2 D$ polynomial surface of order $\varnothing$. is

$$
N_{p}=\frac{1}{2}(\varnothing+1)(\varnothing+2)+r
$$

where $r$ is the number of additional redundant points [5]. If $r=0$ then a surface is constructed that passes precisely through every point. When applied to real data, however, surfaces generated with $r=0$ have a tendency to be excessively noisy, especially the derivative fields. In the present study $r=200$ and the relative importance of these additional points are weighted by a Gaussian function

$$
e^{-\left(\mathbf{L}_{\mathrm{C} G \rightarrow \mathrm{F} / / K)^{2}}\right.}
$$

where $\mathrm{L}_{\mathrm{BG}-\mathrm{FV}}$ is the distance between each interpolation point on the BiGlobal grid, and each data point on the FV/FE grid. $k$ is a smoothness parameter that when increased, creates smoother surfaces due to more of the small scale structures being filtered out. There are consequently three parameters that control the definition of the surfaces, the polynomial order $\varnothing$. the number of additional points $r$, and $\kappa$.

The most appropriate interpolation parameters are determined by searching for those that best reconstruct the previously introduced test function. The test function is applied in the physical co-ordinates to both the FV/FE grid (producing $\mathbf{u}^{\mathrm{FV}}$ and $\partial \mathbf{u}^{\mathrm{FV}} / \partial \mathbf{x}$ ) and the BiGlobal grid (producing $\mathbf{u}^{\mathrm{B} G}$ and $\partial \mathbf{u}^{\mathrm{BC}} / \partial \mathbf{x}$ ). The fields are then mapped into the curvilinear coordinate system for both the FV/FE grid (producing $\xi^{\mathrm{FV}}$ and $\partial \xi^{\mathrm{FV}} / \partial \zeta$ ) and the BiGlobal grid (producing $\xi^{\mathrm{BG}}, \partial \xi^{\mathrm{BG}} / \partial \zeta$ ). In the curvilinear space, the FV/FE test function data $\left(\xi^{\mathrm{F}}\right)$ is then interpolated onto the BiGlobal grid to produce $\xi^{\mathrm{BG} \text {,inr }}$ and spatially differen-

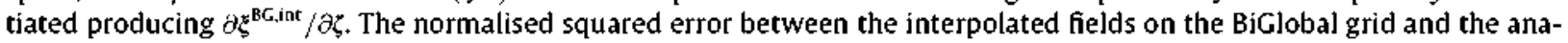
lytical fields mapped directly from the test function applied to the BiGlobal grid in physical space are

$$
\begin{aligned}
& \epsilon_{i}^{\mathrm{int}}=\frac{\int_{V}\left(\xi_{i}^{\mathrm{BC}}-\xi_{i}^{\mathrm{BG}, \mathrm{int}}\right)^{2} d \xi}{\int_{V}\left(\xi_{i}^{\mathrm{BG}}\right)^{2} d \xi}, \\
& \epsilon_{i j}^{\mathrm{int}}=\frac{\int_{V}\left(\partial \xi_{i}^{\mathrm{BG}} / \partial \zeta_{j}-\partial \xi_{i}^{\mathrm{BG}, \mathrm{int}} / \partial \zeta_{j}\right)^{2} d \xi}{\int_{V}\left(\partial \xi_{i}^{\mathrm{BG}} / \partial \zeta_{j}\right)^{2} d \xi} .
\end{aligned}
$$

The parameter set that minimises these error measures for the test function will be adopted for the interpolation of the real data. 


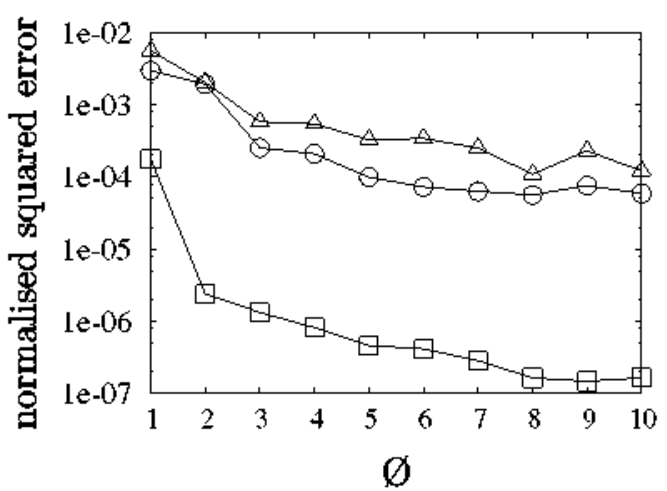

(a)

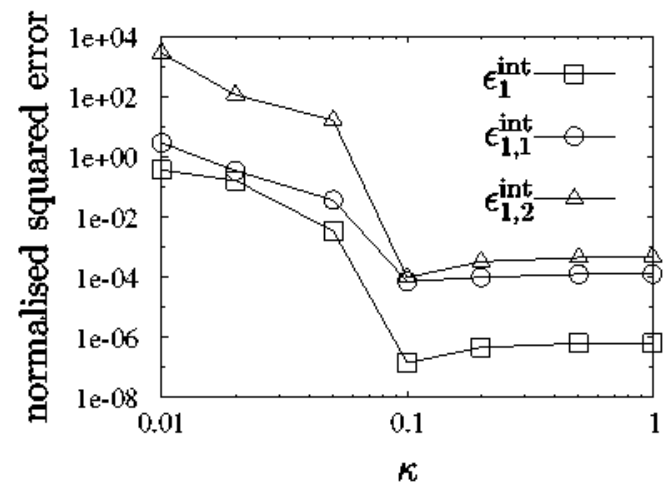

(b)

Fig. 7. The normalised squared error between the test function values evaluated on the BiGlobal grid, and the interpolated values from the finite volume grid to the BiGlobal grid in the curvilinear coordinate system as a function of (a) $\varnothing$ for $\kappa=0.2 ;$ and $(b) x$ for $\varnothing=5$. Note $r=200$ in both cases. The key in (b) is applicable to both figures.

The polynomial order $\varnothing$ has the largest effect on interpolated values and is tested first with $\hbar=0.2$ and $r=200$ in all cases. Fig. $7($ a) illustrates an initial rapid reduction of the error for each component and then begins to level out at $\varnothing=5$. The levelling out is indicative of the polynomial surface now having enough flexibility to adequately fit to the data. The accuracy of the fitted surface may continue to improve with increased polynomial order, but care must be taken when representing the derivative fields. High order polynomial surfaces can produce erratic derivative fields. This is observed from the test function results with the derivative error measures for $\varnothing=9$ greater than $\varnothing=8$. For this reason it is advisable to keep the order of the polynomial as small as possible. From this point forward $\varnothing=5$ has been adopted as it is sufficiently removed from the initial region of significant error.

The $\kappa$ space is tested next with $\varnothing=5$. The stability of the interpolation stencils is directly proportional to $r$ [5]. An unstable stencil is very sensitive to small changes in the stencil values. If $r$ is large and the stencils are stable, $k$ controls the importance of the points within the stencil and $r$ has little effect. The smaller the value of $\kappa$ the more emphasis is placed on the closer surrounding points and perhaps better represents the local gradients. The surface and its derivatives, however, also becomes the less smooth. Fig. 7 (b) illustrates that for $k<0.1$ significantly higher error measures are obtained than for $\kappa>0.1$. To avoid this steep increase in error $k=0.2$ is selected for all subsequent interpolations. To confirm the weak dependence on $r$, a final interpolation test was performed with the parameter set $\varnothing=5, \kappa=0.2$ and $r=800$ and negligible change was observed in the resulting surface. The interpolation parameters $\varnothing=5, k=0.2$ and $r=200$ were initially used for the interpolation of the real data, but in some regions in the domain the derivative fields were not as smooth as required. Increasing the redundancy to $r=800$ solved this problem.

\section{Stability analysis}

The results are now presented for the stability analysis of the steady base flows of the ellipse and an airfoil at $R e_{c}=200$, with a spanwise perturbation wavenumber of $\beta=1$ in physical space. A Krylov subspace dimension of 800 is used in all instances. The eigenvector velocity components are normalised by the largest of the maximum absolute amplitudes of $\hat{u}, \hat{v}$ and $\grave{w}$. The two dominant components are illustrated for each mode below.

\subsection{Ellipse stability resuits}

The ellipse eigen-spectrum is illustrated in Fig. 8(a) with two eigenmodes highlighted: a monotonically growing mode $\mathscr{H}_{e}^{l}$; and an oscillatory decaying mode $\omega_{e}^{l}$. The wall normal domain size for this calculation is $\zeta_{2}^{\max }=11 \mathrm{c}$ and the BiGlobal grid has a number of Fourier collocation points of $N_{\zeta_{1}}=261$, and a number of Chebyshev collocation points of $N_{\zeta_{2}}=180$. Additional lower resolution cases were also run to confirm the convergence of the spectra. The eigenvalues of $\mathscr{H}_{e}^{\dagger}$ and $\sigma_{e} !$ are listed in Fig. 8(b) with the number of cells in each BiGlobal grid and memory required for each calculation. This table illustrates a convergence of the eigenvalues with increased resolution, except perhaps for the growth rate (imaginary component) of the $\mathscr{H}_{e}^{1}$ mode.

The temporal and spatial properties of the modes $\mathscr{A l}_{e}^{\dagger}$ and $\mathscr{b}_{e}^{\dagger}$ from the highest resolution case are now presented. Mode $\mathscr{H}{ }_{e}^{\dagger}$ has an eigenvalue of $\Omega=0.3716 \mathrm{i}$. It is temporally unstable because the imaginary component $\Omega_{\mathrm{i}}>0$ and the amplitude evolves monotonically in time because the real component $\Omega_{\tau}=0$. The eigenvector component $\hat{w}$ is normalised to have a maximum amplitude of unity, which results in ì having an amplitude of order 0.1 , and $\hat{v}$ an amplitude of order 0.01 . $\dot{w}$ is illustrated in Fig. 9(b), and indicates the presence of a large structure starting at the trailing edge, which grows in size 
and amplitude in the streamwise direction. $\hat{u}$ in Fig. 9(a) indicates the presence of a similar elongated structure, this time starting at the leading edge and of the opposite sign as the structure observed in the $\hat{w}$ component. The amplitude of the structure in the $\hat{u}$ component also grows in the streamwise direction. $\mathscr{M}_{e}^{\uparrow}$ has similar features to the "shift" mode of a cylinder wake first discussed in [24]. In that study the "shift" mode described the deformation of the time averaged mean field as the cylinder flow transitioned from a steady to an unsteady state. Mode $\hat{O}_{e}^{\perp}$ has an eigenvalue of $\Omega=2.226-0.0163 \mathrm{i}$. It is temporally stable because $\Omega_{\mathrm{i}}<0$ and the amplitude of this mode oscillates as it decays in time. The $\hat{u}$ and $\hat{v}$ eigenvector fields have maximum amplitudes an order of magnitude higher than that of the $\hat{w}$ field. These fields are illustrated in Fig. 10(a) and (b), respectively. These modes exhibit the classical features of what one would expect for a wake type mode as presented in [25].

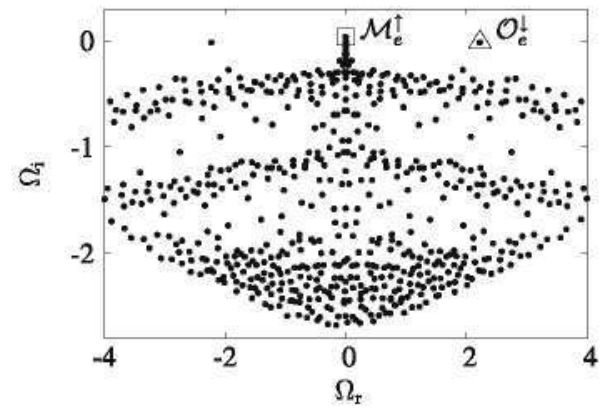

(a)

\begin{tabular}{c|c|c|c|c|c}
$N_{\zeta_{1}}$ & $N_{\zeta_{2}}$ & $\begin{array}{c}\text { Number } \\
\text { of points }\end{array}$ & $\mathcal{M}_{e}^{\dagger}$ & $\mathcal{O}_{e}^{\downarrow}$ & $\begin{array}{c}\text { Memory } \\
(\mathrm{Gb})\end{array}$ \\
\hline 201 & 150 & 30502 & $0.0000+0.3844 \mathrm{i}$ & $2.2249-0.0236 \mathrm{i}$ & 238 \\
231 & 180 & 41992 & $0.0000+0.4100 \mathrm{i}$ & $2.2248-0.0163 \mathrm{i}$ & 452 \\
261 & 180 & 47422 & $0.0000+0.3716 \mathrm{i}$ & $2.2261-0.0163 \mathrm{i}$ & 576
\end{tabular}

(b)

Fig. 8. BiGlobal eigenvalues for the ellipse base flow at $R e_{c}=200$ with $\beta=1$. The (a) complete eigenspectrum with the monotonically growing mode $\mathscr{A l} t_{\mathrm{e}}$ and oscillatory decaying mode $0_{e}^{\downarrow}$ highlighted; and (b) the convergence of eigenvalues $\mathscr{A}_{e}^{\dagger}$ and $\omega_{e}^{\downarrow}$ with the number of points which is $\left(N_{\zeta_{1}}+1\right) \times\left(N_{\zeta 2}+1\right)$.

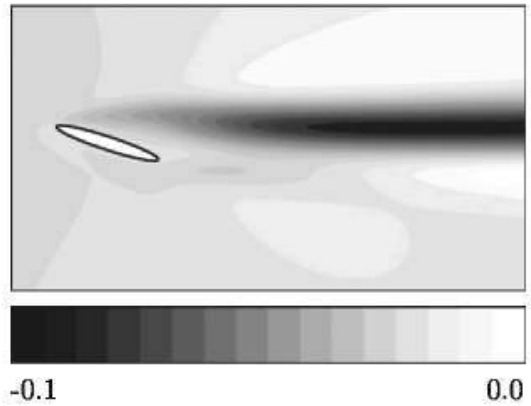

(a)

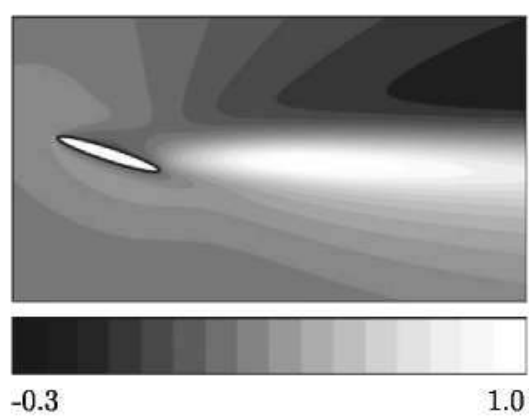

(b)

Fig. 9. Monotonically growing ellipse mode $\mathscr{A}_{e}$. The real component of: (a) $\hat{u}$; and (b) $\hat{w}$. The associated eigenvalue is $\Omega=0.3716 i$.

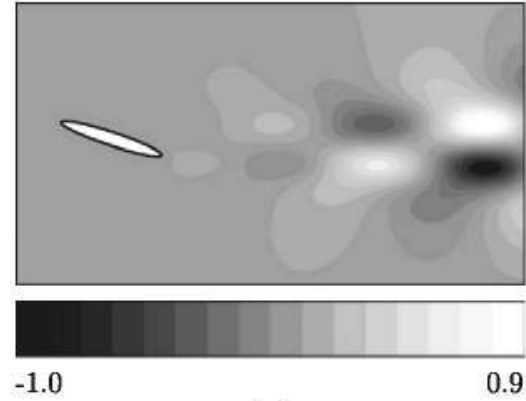

(a)

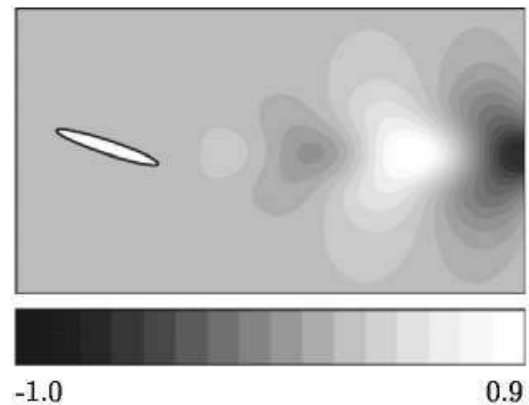

(b)

Fig. 10. Oscillatory decaying ellipse mode $\theta_{\mathrm{e}}^{\dagger}$. The real component of: (a) $\hat{u}$; and (b) $\hat{\nu}$. The associated eigenvalue is $\Omega=-2.226-0.0163 \mathrm{i}$. 


\subsection{Airfoil stability results}

The airfoil eigen-spectrum is illustrated in Fig. 11(a), again with two eigenmodes highlighted: a monotonically growing mode $\mathscr{M}_{a}^{\dagger}$; and an oscillatory growing mode $\varrho_{a}^{\dagger}$. To ensure the BiGlobal grid in physical space is the same size as the previous ellipse case, a larger wall normal domain size of $\zeta_{2}^{\max }=16 \mathrm{c}$ is required. This is due to the properties of the airfoil conformal mapping functions. Having a larger domain means that more points are required to attain the same level of resolution. The highest resolution case has $N_{\zeta_{1}}=249$ and $N_{\zeta_{2}}=250$. At this resolution a minimum of 1024 and 2048 CPUs were respectively needed on the Mare Nostrum and Blue Gene/P facilities in order to allocate the $1 \mathrm{~Tb}$ of memory required to solve the EVP. Note that each node of the former facility has double the amount of memory as those on the latter. As was done previously for the ellipse case, additional lower resolution cases were run to confirm the convergence of the spectra. The eigenvalues of $\mathscr{M}_{a}^{\dagger}$ and

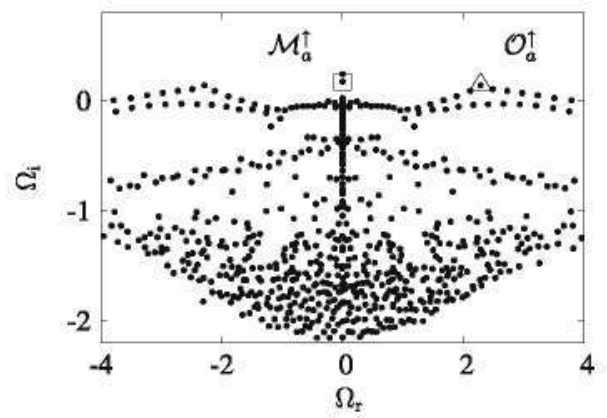

(a)

\begin{tabular}{c|c|c|c|c|c}
$N_{\zeta_{1}}$ & $N_{\zeta_{2}}$ & $\begin{array}{c}\text { Number } \\
\text { of points }\end{array}$ & $\mathcal{M}_{a}^{\dagger}$ & $\mathcal{O}_{a}^{\dagger}$ & $\begin{array}{c}\text { Memory } \\
(\mathrm{Gb})\end{array}$ \\
\hline 201 & 128 & 26058 & $0.0000+0.1548 \mathrm{i}$ & $2.2179+0.1396 \mathrm{i}$ & 72 \\
201 & 200 & 40602 & $0.0000+0.1727 \mathrm{i}$ & $2.2213+0.1703 \mathrm{i}$ & 422 \\
249 & 250 & 62750 & $0.0000+0.1725 \mathrm{i}$ & $2.2883+0.1664 \mathrm{i}$ & 1008
\end{tabular}

(b)

Fig. 11. BiGlobal eigenvalues for the airfoil base flow at $\operatorname{Re}_{c}=200$ with $\beta=1$. The (a) complete eigenspectrum with the monotonically growing mode $\mathscr{A}_{a}^{\dagger}$ and oscillatory decaying mode $\hat{O}_{a}^{\uparrow}$ highlighted; and (b) the convergence of eigenvalues $\mathscr{M}_{a}^{\dagger}$ and $\hat{O}_{a}^{\dagger}$ with the number of points which is $\left(N_{\zeta_{1}}+1\right) \times\left(N_{\zeta_{2}}+1\right)$.

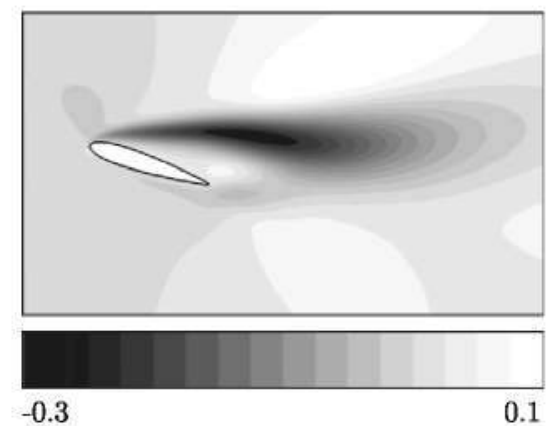

(a)

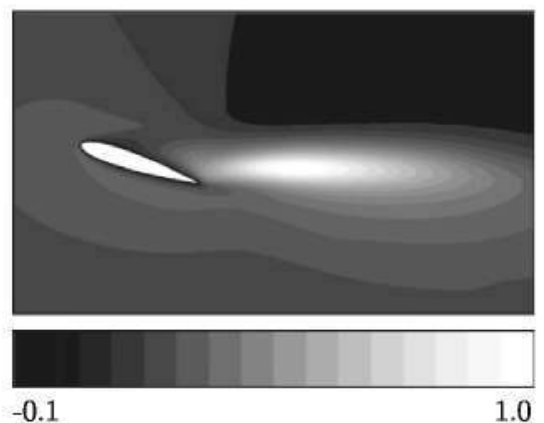

(b)

Fig. 12. Monotonically growing airfoil mode $\mathscr{A}_{a}^{\dagger}$. The real component of: (a) $\hat{u}$; and (b) $\hat{w}$. The associated eigenvalue is $\Omega=0.1663 \mathrm{i}$.

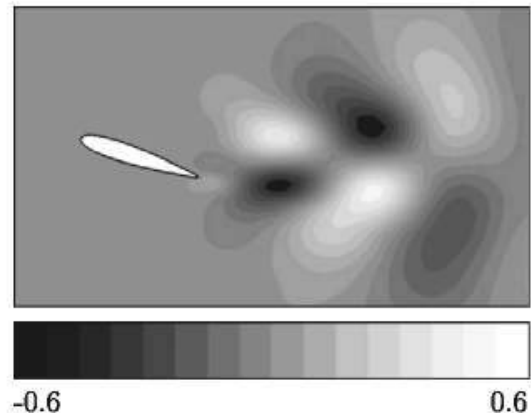

(a)

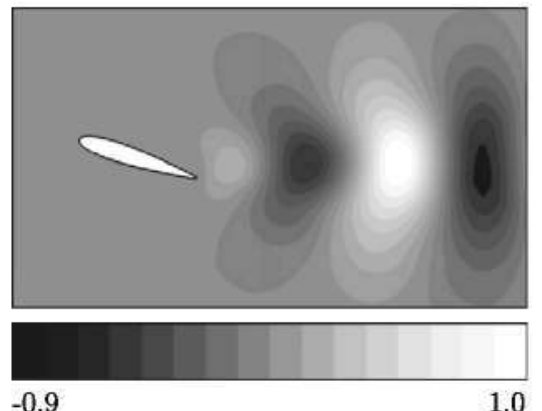

(b)

Fig. 13. Oscillatory growing airfoil mode $\hat{O}_{a}^{\dagger}$. The real component of: (a) $\hat{u}$; and (b) $\hat{\nu}$. The associated eigenvalue is $\Omega=2.3991+0.2019 \mathrm{i}$. 
$\varrho_{a}^{1}$ are listed in Fig. 11(b). Convergence of the eigenvalues is again demonstrated. It is not clear, however, whether the growth rate of the $\omega_{a}^{l}$ mode is converged at this resolution.

The properties of the eigenmodes $\mathscr{H}_{a}^{l}$ and $\mathscr{O}_{a}^{1}$ from the highest resolution case are again presented. Mode $\mathscr{H}_{\mathrm{a}}^{l}$ is a monotonically unstable mode with an eigenvalue of $\Omega=0.1725$ i. Its eigenvector fields $\hat{u}$ and $\hat{w}$ are illustrated in Fig. 12 (a) and (b), respectively. This mode has very similar characteristics to the $\mathscr{H}_{e}^{1}$ mode for the ellipse, with the structures growing in size and amplitude in the streamwise direction. Mode $\omega_{a}^{\Gamma}$ has an eigenvalue of $\Omega=2.2883+0.1664$. The eigenvector fields of $\omega_{\sigma}^{\text {I }}$ in Fig. 13 again have the classical wake type mode characteristics observed in the ellipse mode $\omega_{e}^{l}$. However, for this particular value of $\beta$ the airfoil mode $\sigma_{a}^{\dagger}$ is unstable whilst the ellipse mode $\sigma_{e}^{j}$ is stable. Additional simulations of the airfoil basic state were undertaken, and it was found that the flow is unsteady at $R e_{c}=225$. This indicates that the flow is in a very sensitive region in $\operatorname{Re}_{c}$ space.

\section{Concluding remarks}

A novel methodology has been presented to analyse the problem at hand in the frame of BiGlobal stability theory. Conformal mapping techniques were used to transform a rectangular computational mesh in order to represent the geometry of the problem. The Navier-Stokes equations written in curvilinear coordinates were linearised and the linear operator for the BiGlobal eigenvalue problem obtained. Two similar geometries were analysed: a NACA0015 airfoil; and an ellipse with $A_{R}=8$. Both configurations were at the same angle of attack of $18^{\circ}$ and $R e_{\mathrm{r}}=200$. At these conditions both flows were steady and separation occurred just downstream of the leading edge. The amplitude functions of the eigenmodes recovered were related to the wake and the separated regions.

The question may be raised regarding the computing cost of, on the one hand setting up the discrete matrix and computing its leading Ritz values and corresponding vectors and, on the other hand, employing time-marching schemes in which the matrix is never formed (eg. [2]). Indeed, the leading wake mode in the related application of a NACA 0012 airfoil [38] has been obtained by the latter approach at orders-of-magnitude lower computing effort compared with that resulting from the methodology presented herein. However, no direct comparisons have been performed, in which a fixed, large $\left(O\left(10^{3}\right)\right)$ number of eigenvalues and ejgenvectors need be computed by the time-stepping approach, and no speculations will be attempted on the potential existence of thresholds beyond which one or the other method should be used. What has been shown in earlier work [30] and is confirmed by the present computations is that storage and parallel inversion of the matrix is a process which scales linearly with the number of processes utilised, such that access to an increasingly large window of the full eigenspectrum may be offered by massive parallelisation. Nevertheless, the question whether the proposed storageand-inversion methodology is a viable alternative to the very efficient time-stepping approaches available in the community is a question which should be addressed carefully in future works.

\section{Acknowledgments}

The first author would like to acknowledge the Égide group for funding his research stay in Europe via the Eiffel Doctorate grant. The second and third authors were sponsored by the Air Force Office of Scientific Research, Air Force Material Command, USAF, under Grant number FA8655-06-1-3066 to nu modelling s.l, entitled Global instabilities in laminar separation bubbles. The Grant is monitored by Dr. D. Smith of AFOSR (originally by Lt. Col. Dr. Rhett Jefferies) and Dr. S. Surampudi of EOARD. The views and conclusions contained herein are those of the author and should not be interpreted as necessarily representing the official policies or endorsements, either expressed or implied, of the Air Force office of Scientific Research or the US Government. Computations have been performed on the CeSViMa (http:/www.cesvima.upm.es), MareNostrum (http:/www.bsc.es), and Blue-Gene/P (Forschungzentrum Juelich - http:/www.fz-juelich.de) facilities. The authors would also like to acknowledge the Stanford Center for Turbulence Research for the use of CDP code in obtaining the airfoil basic flows, and Dr. L. González for the use of the ADFC code (http:/www.sourceforge.net/adfc) in obtaining ellipse basic flow results.

\section{Appendix A. Perturbation form of the Navier-Stokes equations in curvilinear coordinates}

The linearised perturbation form of the NSE in curvilinear coordinates is fully expanded below. A 2D structure to the base flow is assumed where $\bar{\zeta}_{3}=\partial \bar{\xi}_{3} / \partial \xi_{j}=\partial \bar{\zeta}_{i} / \partial \dot{\xi}_{3}=0$. Also as the conformal mappings are only applied in the $(x, y)$ plane, $z=\zeta_{3}$. This implies that $\partial z / \partial r_{3}=\partial s_{3}^{*} / \partial z=1, h_{3}=1$ and all derivatives of $h_{3}$ are zero. Applying these simplifications the continuity equation is

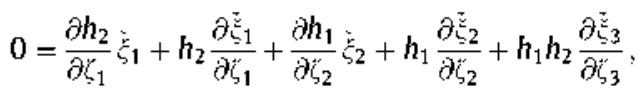

the momentum equation for $\tilde{\xi}_{1}$ is

$$
\frac{\partial \vec{\xi}_{1}}{\partial t}=-\frac{1}{h_{1}} \frac{\partial \bar{\xi}_{1}}{\partial \xi_{1}} \dot{\xi}_{1}-\frac{1}{h_{2}} \frac{\partial \bar{\xi}_{1}}{\partial \xi_{2}} \vec{\xi}_{2}-\frac{1}{h_{1}} \frac{\partial \overrightarrow{\mathrm{p}}}{\partial \xi_{1}}-Z_{2 D} \dot{\xi}_{1},
$$


the momentum equation for $\vec{\xi}_{2}$ is

$$
\frac{\partial \tilde{\xi}_{2}}{\partial \mathrm{t}}=-\frac{1}{h_{1}} \frac{\partial \bar{\xi}_{2}}{\partial \tilde{\zeta}_{1}} \tilde{\xi}_{1}-\frac{1}{h_{2}} \frac{\partial \bar{\xi}_{2}}{\partial \zeta_{2}} \dot{\xi}_{2}-\frac{1}{h_{2}} \frac{\partial \dot{p}}{\partial \zeta_{2}}-Z_{2 D} \tilde{\tilde{\xi}}_{2}, \quad \text { and }
$$

the momentum equation for $\hat{\xi}_{3}$ is

$$
\frac{\partial \vec{\xi}_{3}}{\partial t}=-\frac{\partial \vec{p}}{\partial \dot{\zeta}_{3}}-Z_{2 D} \vec{\xi}_{3},
$$

where $Z_{2 D}$ is a linear operator given by

$$
\begin{aligned}
Z_{2 D}= & \bar{\zeta}_{1} \frac{1}{h_{1}} \frac{\partial}{\partial \zeta_{1}}+\bar{\xi}_{2} \frac{1}{h_{2}} \frac{\partial}{\partial \zeta_{2}} \\
& -\frac{1}{\operatorname{Re} h_{1} h_{2}}\left[\frac{\partial h_{2}}{\partial \zeta_{1}} \frac{1}{h_{1}} \frac{\partial}{\partial \zeta_{1}}-\frac{\partial h_{1}}{\partial \zeta_{1}} \frac{h_{2}}{h_{1}^{2}} \frac{\partial}{\partial \zeta_{1}}+\frac{h_{2}}{h_{1}} \frac{\partial^{2}}{\partial \zeta_{1}^{2}}+\frac{\partial h_{1}}{\partial \zeta_{2}} \frac{1}{h_{2}} \frac{\partial}{\partial \zeta_{2}}-\frac{\partial h_{2}}{\partial \zeta_{2}} \frac{h_{1}}{h_{2}^{2}} \frac{\partial}{\partial \zeta_{2}}+\frac{h_{1}}{h_{2}} \frac{\partial^{2}}{\partial \zeta_{2}^{2}}+h_{1} h_{2} \frac{\partial^{2}}{\partial \zeta_{3}^{2}}\right] .
\end{aligned}
$$

\section{Appendix B. Linear operator in curvilinear coordinates}

Here the equations presented in Appendix $A$ are presented in a matrix form representative of the means that the EVP is posed in the code. A complementary 2D perturbation structure is now also assumed. It has a periodic structure in time with a complex frequency $\Omega$, and a periodic structure in the spanwise direction with real wavenumber $\beta$. Applying this perturbation structure to the equations presented Appendix $A$, the system can be written in matrix form as

$$
\begin{aligned}
& \mathbf{A}(\overline{\mathbf{q}}, \beta) \mathbf{q}=-\mathrm{i} \Omega \text { B̀q }, \quad \text { where }
\end{aligned}
$$

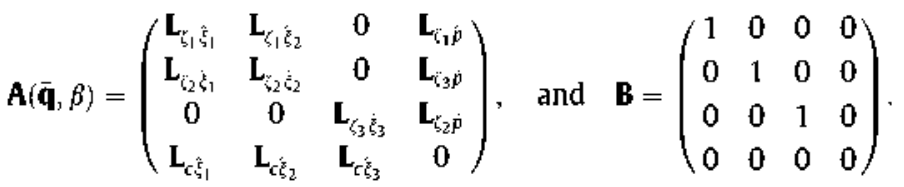

The blocks of linear operator $\mathbf{A}(\overline{\mathbf{q}}, \beta)$ are detailed below. Note in the following equations the notation of $\partial_{\zeta_{j}} \equiv \partial / \partial \zeta_{i}$ and $\partial_{\zeta \zeta_{j}} \equiv \partial^{2} / \partial \zeta_{i} \partial \zeta_{j}$ is adopted. The blocks associated with the continuity equation are

$$
\begin{aligned}
& \mathbf{L}_{c \xi_{1}}=h_{2} \partial_{c_{1}}+\frac{\partial h_{2}}{\partial \xi_{1}}, \\
& \mathbf{L}_{c \xi_{2}}=h_{1} \partial_{c_{2}}+\frac{\partial h_{1}}{\partial \xi_{2}}, \text { and } \\
& \mathbf{L}_{c \xi_{3}}=\mathrm{i} \beta h_{1} h_{2} .
\end{aligned}
$$

The blocks associated with the momentum equation for $\hat{\xi}_{1}$ are

$$
\begin{aligned}
& \mathbf{L}_{\xi_{1} \dot{\zeta}_{1}}=-\frac{1}{h_{1}} \frac{\partial \bar{\xi}_{1}}{\partial \zeta_{1}}-\mathbf{Z}_{2 D}, \\
& \mathbf{L}_{\zeta_{1} \xi_{2}}=-\frac{1}{h_{2}} \frac{\partial \bar{\xi}_{1}}{\partial \zeta_{2}}, \text { and } \\
& \mathbf{L}_{\zeta_{1} \hat{p}}=-\frac{1}{h_{1}} \partial_{\zeta_{1}} .
\end{aligned}
$$

The blocks associated with the momentum equation for $\vec{\xi}_{2}$ are

$$
\begin{aligned}
& \mathbf{L}_{\gamma_{2} \dot{\xi}_{1}}=-\frac{1}{h_{1}} \frac{\partial \bar{\xi}_{2}}{\partial \xi_{1}}, \\
& \mathbf{L}_{\gamma_{2} \dot{\sigma}_{2}}=-\frac{1}{h_{2}} \frac{\partial \bar{\xi}_{2}}{\partial \xi_{2}}-\mathbf{z}_{2 D}, \text { and } \\
& \mathbf{L}_{\zeta_{2} p}=-\frac{1}{h_{2}} \partial_{\xi_{2}} .
\end{aligned}
$$

Finally, the blocks associated with the momentum equation for $\bar{\xi}_{3}$ are 


$$
\begin{aligned}
& \mathbf{L}_{\mho_{3} \dot{\xi_{3}}}=-\mathbf{Z}_{2 D}, \text { and } \\
& \mathbf{L}_{\xi_{3} \hat{p}}=-\mathbf{i} \beta .
\end{aligned}
$$

where $Z_{2 D}$ is the discrete form of $Z_{2 D}$ and given by

$$
\begin{aligned}
\mathbf{Z}_{2 D}= & {\left[\frac{\bar{\xi}_{1}}{h_{1}}-\frac{1}{\operatorname{Re}}\left(\frac{\partial h_{2}}{\partial \zeta_{1}} \frac{1}{h_{1}^{2} h_{2}}-\frac{\partial h_{1}}{\partial \zeta_{1}} \frac{1}{h_{1}^{3}}\right)\right] \partial_{\zeta_{1}}-\frac{1}{\operatorname{Re}_{1}^{2}} \partial_{\zeta_{1}} \partial_{\zeta_{1}}+\left[\frac{\bar{\xi}_{2}}{h_{2}}-\frac{1}{\operatorname{Re}}\left(\frac{\partial h_{1}}{\partial \zeta_{2}} \frac{1}{h_{1} h_{2}^{2}}-\frac{\partial h_{2}}{\partial \zeta_{2}} \frac{1}{h_{2}^{3}}\right)\right] \partial_{\zeta_{2}}-\frac{1}{\operatorname{Reh}_{2}^{2}} \partial_{\zeta_{2}} \partial_{\zeta_{2}} } \\
& +\frac{\beta^{2}}{\operatorname{Re}}
\end{aligned}
$$

\section{References}

[1] E. Ákervik, J. Heepfner, U. Elırenstein. D. Henningson, Optimal growth, model reduction and control in a separated boundary-layer flow using global eigenmodes, J. Fluid Mech 579 (2007) 305-314.

[2] D. Barkley. H. Blackburn, S. Sherwin, Direct optimal growth analysis for timesteppers, Int. J. Numer. Meth. Fluids 57 (9) (2008) $1435-1458$.

[3] C. Canuto, M. Hussaini, A. Quarteroni, T. Zang. Spectral Methods: Fundamentals in Single Domains, Springer, 2006.

[4] F. Chedevergne, G. Casalis, T. Féraillec, BiGlobal linear stability analysis of the flow induced by wall injection, Phys. Fluids 18 (1) (2006) 014103:1014103:14.

[5] S. Chenoweth, J. Soria, A. Ooi, A singularity-avoiding moving least squares scheme for two dimensional unstructured meshes, J. Comput. Phys. 228 (15) (2009) 5592-5619.

[6] J.-M. Chomaz, Global instabilities in spatially developing flows: non-normality and nonlinearity. Ann. Rev. Fuid. Mech. 37 (2005) $357-392$.

[7] J. Crouch, A. Gabaruk, D. Magidov, A. Travin, Origin of transonic buffet on aerofoils, J. Fluid Mech. 628 (2009) 357-369.

[8] J.D. Crouch, A. Garbaruk, D. Magidov, Predicting the onset of flow unsteadiness based on global instability. J. Comput. Phys. 224 (2007) 924-940.

[9] P.G. Drazin, W.H. Reid, Hydrodynamic Stability, Cambridge University Press, 2004.

[10] U. Ehrenstein. F. Gallaire. Two-dimensional global low-frequency oscillations in a separating boundary-layer flow, J. Fuid Mech. 614 (2008) 315-327.

[11] N. Fiétier, M. Deville, Time-dependent algorithms for the simulation of viscoelastic flows with spectral element methods: applications and stability. J. Comput. Phys. 186 (1) (2003) 93-121.

[12] F. Giannetti, P. luchini, Structural sensitivity of the first instability of the cylinder wake. J. Fuid Mech. 581 (2007) 167-197.

[13] L. González, R. Bermejo, A semi-Lagrangian level set method for incompressible Navier-Stokes equations with free surface, Int. J. Numer. Meth. Fluids 49 (2005) 1111-1146.

[14] G. Karniadakis, 5. Sherwin, 5pectral thp element methods for Computational Fluid Dynamics, second ed., Oxford University Press, 2005.

[15] J. Kim, T.R. Bewley, A linear systems approach to flow control, Ann. Rev. Fluid. Mech. 39 (2007) 373-417.

[16] V. Kitsios, D. Rodriguez, V. Theofilis, A. Ooi. J. Soria, BiGlobal instability analysis of turbulent flow over an airfoil at an angle of attack, in: 38 th Fluid Mechanics Conference and Exhibit, AlAA-Paper 2008-4384, 2008.

[17] R.-S. Lin, M.R. Malik, On the stability of attachment-line boundary layers. Part 1. The incompressible swept Hiemenz flow, J. Fluid Mech. 311 (1996) 239-255.

[18] R.-S. Lin, M.R. Malik, On the stability of attachment-line boundary layers. Part 2. The effect of leading-edge curvature, J. Fluid Mech. 333 (1996) 125137.

[19] F. Longueteau, J.-P. Brazier, BiGlobal stability computations on curvilinear meshes, C. R. Mécanique 336 (11-12) (2008) $828-834$.

[20] M. Macaraeg, C. Streett, M. Hussaini, A spectral collocation solution to the compressible stability eigenvalue problem, Tech. Rep. TP-2858, NASA, 1988.

121] K. Mahesh, G. Constantinescu, P. Moin, A numerical method for large Eddy simulations in complex geometries, J. Comput. Phys. 197 (2004) 215-240.

222] M. Malik, Numerical methods for hypersonic boundary layer stability, J. Comp. Phys. 86 (2) (1990) 376-413.

[23] P. Morse. H. Feshbach, Methods of Theoretical Physics. Part 1. Feshbach Publishing. 1953.

[24] B. Noack, K. Afanasiev, M. Morzynski, G. Tadmor, F. Thiele, A hierarchy of low dimensional models for the transient and post transient cylinder wake, J. Fluid Mech. 497 (2003) 335-363.

[25] B. Noack, H. Eckelmann, A global stability analysis of the steady and periodic cylinder wake. J. Fluid Mech. 270 (1994) $297-330$.

[26] B. Noack, M. Schlegel, B. Ahlborn, G. Mutschke, M. Morzyński, P. Compte, G. Tadmor, Finite time themodynamics of unsteady fluid flows, J. Non-Egui. Thermo. 33 (2008) $103-148$.

[27] 5. Orszag. Accurate solution of the Orr-5ommerfeld stability equation. J. Fluid Mecli. 50 (1971) 689-703.

[28] R. Peyret, Spectral Methods for Incompressible Viscous Flow, Springer, 2002.

129] E. Piot. G. Casalis, U. Rist. Stability of the laminar boundary layer flow encountering a row of roughness elements: BiGlobal stability approach and DN5. Eur. J. Mech. B - Fluids 27 (6) (2008) 684-706.

[30] D. Rodriguez, V. Theofilis, Massively parallel numerical solution of the BiGlobal linear instability eigenvalue problem using dense linear algebra, AlAA J, 2009, in press.

[31] Y. Saad, Numerical Methods for Large Eigenvalue Problems, Algorithms and Architectures for Advanced Scientific Computing, Manchester University Press, 1992.

[32] P.J. Schmid, Nonmodal stability theory, Ann. Rev. Fluid Mech. 39 (2007) 129-162.

[33] P.J. Schmid, D.S. Henningson, Stability and Transition in Shear Flows, Springer-Verlag, 2001.

[34] 5.J. Sherwin. H.M. Blackburn. Three-dimensional instabilities and transition of steady and pulsatile axisymmetric stenotic flows. J. Fluid Mech. 533 (2005) 297-327.

[35] T. Tatsumi. T. Yoshimura, 5tability of the laminar flow in a rectangular duct. J. Fluid Mech. 212 (1990) 437-449.

136] V. Theofilis, Advances in global linear instability analysis of nonparallel and three-dimensional flows. Prog. Aero. $5 \mathrm{ci}, 39$ (2003) $249-315$.

[37] V. Theofilis, The role of instability theory in flow control, in: R.D. Joslin, D. Miller (Eds.), Fundamentals and Applications of Modern Flow Control AlAA Progress in Aeronautics and Astronautics, vol, 231, 2009, pp. 73-116.

[38] V. Theofilis, D. Barkley, S.]. Sherwin, Spectralfhp element technology for flow instability and control, Aero. J. 106 (1065) (2002) $619-625$.

[39] V. Theofilis, 5. Hein, U. Dallmann, On the origins of unsteadiness and three-dimensionality in a laminar separation bubble, Phil. Trans. Roy. Soc. Lond. A $358(1777)(2000) 3229-3246$. 